\title{
ABBREVIATIONS OF WORKS BY LEVINAS
}

AE Autrement qu'être ou au-delà de l'essence. The Hague: Martinus Nijhoff, 1974.

BPW Emmanuel Levinas: Basic Philosophical Writings. Edited by Adriaan T. Peperzak, Simon Critchley, and Robert Bernasconi. Translated by the editors et al. Bloomington: Indiana University Press, 1996.

CPP Collected Philosophical Papers. Translated by Alphonso Lingis. The Hague: Martinus Nijhoff, 1974.

D "Discussion of Existentialism by Nicolas Berdiaeff, Maurice de Gandillac, Georges Gurvitch, Alexandre Koyré, Emmanuel Levinas, Gabriel Marcel." In Jean Wahl, $A$ Short History of Existentialism, 35-56. New York: Philosophical Library, 1949.

DE De l'évasion. Introduction and notes by Jacques Rolland. Montpellier: Fata Morgana, 1982.

DEE De l'existence à l'existant. Paris: Vrin, 1947.

DEH Discovering Existence with Husserl. Translated and edited by Richard A. Cohen and Michael B. Smith. Evanston, Ill.: Northwestern University Press, 1998.

DEHH En découvrant l'existence avec Husserl et Heidegger. 3d ed. Paris: Vrin, 1974.

DF Difficult Freedom. Translated by Seán Hand. Baltimore: Johns Hopkins University Press, 1990.

DL Difficile liberté. 3d ed. Paris: Albin Michel, 1984.

DVI De Dieu qui vient à l'idée. Paris: Vrin, 1982.

EE Existence and Existents. Translated by Alphonso Lingis. The Hague: Martinus Nijhoff, 1978.

EN Entre nous: Essais sur le penser-à-l'autre. Paris: Grasset, 1991.

ENT Entre Nous: On Thinking-of-the-Other. Translated by Mi- 
chael B. Smith and Barbara Harshav. New York: Columbia University Press, 1998.

HAH Humanisme de l'autre homme. Montpellier: Fata Morgana, 1976.

MHO "Martin Heidegger and Ontology." Translated by the Committee of Public Safety. Diacritics 26, no. 1 (1996): 11-32.

NTR Nine Talmudic Readings. Translated by Annette Aronowicz. Bloomington: Indiana University Press, 1990.

OBBE Otherwise than Being, or, Beyond Essence. Translated by Alphonso Lingis. The Hague: Martinus Nijhoff, 1981.

QLT Quatre lectures talmudiques. Paris: Minuit, 1968.

RO “La réalité et son ombre." Les Temps Modernes 38 (1948): 771-89. Translated by Alphonso Lingis as "Reality and Its Shadow," CPP, 1-13.

S "La Substitution," Revue Philosophique de Louvain (1968): 487-508. Translated by Peter Atterton, Simon Critchley, and Graham Noctor as "Substitution," BPW, 80-95.

TA Le temps et l'autre. Montpellier: Fata Morgana, 1979.

TeI Totalité et l'infini: Essai sur l'exteriorité. The Hague: Martinus Nijhoff, 1961.

TI Totality and Infinity: An Essay on Exteriority. Translated by Alphonso Lingis. Pittsburgh: Duquesne University Press, 1969.

TIH The Theory of Intuition in Husserl's Phenomenology. Translated by André Orianne. Evanston, Ill.: Northwestern University Press, 1973.

TIPH Théorie de l'intuition dans la phénomenologie de Husserl. Paris: Alcan, 1930.

TO Time and the Other. Translated by Richard Cohen. Pittsburgh: Duquesne University Press, 1985.

TrO "The Trace of the Other." Translated by Alphonso Lingis. In Deconstruction in Context, edited by Mark C. Taylor, 345-59. Chicago: University of Chicago Press, 1986. 
EMMANUEL LEVINAS 
